\title{
Counting Pig using Marker-Controlled Watershed Segmentation
}

\author{
Salam Jayachitra Devi ${ }^{*}$ and Kh. Manglem Singh ${ }^{2}$ \\ ${ }^{1}$ National Research Centre on Pig, Guwahati, India \\ ${ }^{2}$ National Institute of Technology, Manipur, India \\ *Corresponding author
}

\section{A B S T R A C T}

\section{Keywords}

Image

segmentation, counting pig, Object detection,

Overlapping objects

Article Info

Accepted:

12 December 2020

Available Online:

10 January 2021
Counting the total number of pigs manually on a large-scale pig farm is a crucial and inefficient task. As this process is time-consuming and includes many critical points that can lead to miscalculation. Some of the challenging issues in pig counting include overlapping, partial occlusion, different viewpoint that limits the usage of traditional object detection methods. Image segmentation is used for object detection, which separate foreground and background pixels of the images. In this paper, we used Marker-Controlled Watershed segmentation method for counting pig in an image. Here, different image thresholding techniques such as Otsu threshold, Adaptive threshold and manual threshold is considered. The structural similarity of these thresholding techniques is determined using jaccards coefficient index. Otsu threshold gives the best similarity scores. The average processing time of these thresholding techniques is also determined. Further, the images obtained from Otsu threshold is checked for overlapping objects. In case of image with overlapping objects, the segmentation is done using marker-controlled watershed segmentation algorithm to segregate the overlapping objects and label the objects individually. In case of non overlapping, objects present in the images obtained from Otsu threshold are label directly to count the number of pigs present in the image. Hence, this segmentation process provides an efficient way for counting pigs in an image.

\section{Introduction}

In India pig farming is one of the profitable livestock businesses and most of the farmers are successful in rearing commercial pig business. But rearing of pig is still unorganized endeavor that requires adoption of science and technology to increase production, monitor the livestock, reduce enterprise cost, automate growth management, monitor healthy environmental conditions for pigs which will leads to increase in farmer's profit and reduce manpower $(1,2)$. In large scale pig farming, counting of pig is also one of the challenging issues. Currently, pig counting is very important in large scale pig production management. Counting pig accurately can help farmers in improving pig management by reducing unnecessary loss in feeding, cost reduction in piggery construction etc $(3,4)$.

However, several factors that restrict the accurate counting of pigs are overlapping of 
pigs, illumination changes, camera's perspective, etc (5). Counting manually may lead to missed estimation, time consuming and expensive. Such issues commonly occur in large scale pig farming. There are many computer vision and artificial intelligence techniques that solve the pig management problem effectively (6). Research on segregating foreground and background objects is the basic work of advanced research in artificial intelligence for pig detection, pig behavior recognition, pig tracking, etc $(7,8$, 23). Therefore, image segmentation is the most important steps in object detection which cluster pixels into relevant image regions.

Effective segmentation of foreground objects in a complex environment is still the hot topic and challenging research. There are several background detection algorithm like frame difference, background difference, background subtraction algorithm and mixed Gaussian model which cannot detect foreground objects effectively (9-14). None of the foreground detection algorithm can be used universally across different image types without any drawbacks. Each algorithm has its own limitations and cannot handle all the complex scenarios such as illumination, heavy shadow etc. in a common way (15).

Object tracking algorithm based on initial segmentation and background estimation was developed $(9,15)$. The drawback in this method is it requires to move the foreground target all the time else the target might lose easily. Segmentation algorithm proposed by Perner (10) detects the position of the pigs using threshold segmentation and background subtraction. Further, this was used for pig behavior observation under the effects of drugs. Based on the mixed Gaussian method and 2-D wavelet transform, a target detection method based on background modeling was developed by Gang (13). This method is still invalid for less detection on mobile foreground targets. An algorithm was also developed for monitoring pig behavior using a mobile phone (14). This system can track the pig without losing targets for a few minutes. But the system has problem in identifying objects influence of dust and dirt in a complex environment of piggery.

According to the survey, image segmentation is used to detect pigs and identify the region of interest (16). Detection of foreground targets is not sufficient for accurate object counting in an image. For object counting, we need an efficient algorithm to segregate the overlapping objects as well as remove the noise present in the image. Therefore, for segregating overlapping objects Markercontrolled watershed segmentation is one of an efficient algorithm that have interesting properties which is useful in various image segmentation applications. But in some cases, it also has drawbacks of over segmentation and noise sensitivity. This paper will mainly focus on image segmentation of piggery and counting the total pigs available in the image. For this, Otsu thresholding method and Marker-controlled watershed segmentation is adopted. Meanwhile, morphological method is also adopted to remove the noise from the image. We used contours area to identify the overlapping objects in an image automatically. At last, we used bwlabelling algorithm to label each pig simultaneously.

\section{Materials and Methods}

The segmentation algorithm for countings pigs is a combinational algorithm which consists of image threshold to perform foreground extraction, watershed transformation, marker-controlled watershed segmentation, bwlabelling and counting of pigs in an image. This combinational algorithm also includes morphological processing method in order to simplify the 
image pixels and remove the cracks, grille, and other noise present in the image and established a pixel array of foreground and background which is the input of markercontrolled watershed segmentation algorithm. The flow chart of this algorithm is depicted in figure 1 .

\section{Otsu threshold}

In the field of computer vision and image processing, this method is mainly used for automatic image thresholding which separate image pixels into foreground and background classes. This single intensity threshold is determined either by minimizing intra class intensity variance or maximizing inter class intensity variance. This algorithm checked for threshold exhaustively that can minimize the intra-class variance. The weighted sum of variances for the foreground and background classes can be defined as

$\sigma_{\omega}^{2}(t)=\omega_{0}(t) \sigma_{0}^{2}(t)+\omega_{1}(t) \sigma_{1}^{2}(t)$

Where $\omega_{0}$ and $\omega_{1}$ are the weighted probabilities of the two classes. ${ }^{t}$ denotes the threshold. Variances of the two classes is denoted by $\sigma_{0}^{2}$ and $\sigma_{1}^{2}$. The weighted class probability $\omega_{0}(t)$ and $\omega_{1}(t)$ are computed based on the of ${ }^{\mathcal{L}}$ bins of histogram (5).

$\omega_{0}(t)=\sum_{i=0}^{t-1} p(i)$

$\omega_{1}(t)=\sum_{i=t}^{\mathcal{L}-1} p(i)$

In case of two classes, maximizing inter class variance is equal to minimizing the intra class variance.
Where $\mu_{0}(t), \mu_{1}(t)$ and $\mu_{T}$ denotes the class means and defined as

$\mu_{0}(t)=\sum_{i=0}^{t-1} i p(i) / \omega_{0}(t)$

$\mu_{1}(t)=\sum_{i=t}^{\mathcal{L}-1} i p(i) / \omega_{1}(t)$

$\mu_{T}=\sum_{i=0}^{\mathcal{L}-1} i p(i)$

The class mean and probabilities can be computed recursively. This yields to an effective method.

\section{Morphological processing}

In this paper, we performed morphological operation on a binary image obtained as output from the Otsu threshold to create a new binary image where pixel is assigned with a non-zero value only when the test is successful at a particular location in the given input image.

This consists of structuring element which is a small binary pixel matrix of value either zero or one. Structuring elements play the same role as convolution kernels for image filtering in morphological image processing. Fundamental operations of morphological image processing include Erosion and Dilation $(17,18,24)$.

Erosion operation in binary image $f_{\text {using }}$ structuring element ${ }^{\text {s }}$ generate a new image $\mathrm{g}=\mathrm{f} \ominus{ }^{\mathrm{s}}$ with $\mathrm{g}(\mathrm{x}, \mathrm{y})=1_{\text {if }}{ }^{\mathrm{s}}$ fits $\mathrm{f}$, otherwise 0 and this is repeated for all pixel coordinates. This resulted in the reduction of regions of interest sizes in the binary image. From eroded image, an original image boundaries of each region can also be determined by subtracting the two images. 
Dilation operation in binary image $f$ using structuring element ${ }^{\text {s }}$ generate a new image $\mathrm{g}=\mathrm{f} \oplus{ }^{\mathrm{s}}$ with $\mathrm{g}(x, y)=1$ if ${ }^{\mathrm{s}}$ fits $\mathrm{f}$, otherwise 0 and this is repeated for all pixel coordinates. This is exactly opposite to erosion as it adds pixels layer both to the inner as well as to the outer boundaries of regions.

Here in this paper, we used opening morphological filtering operation. Opening operation of a binary image $f_{\text {using structuring }}$ element ${ }^{s}$ is performing an erosion operation followed by dilation operation defined as fos $=(f \ominus s) \oplus s$. We used this operation to open the gap between connected objects by a bridge of pixels. In this operation structuring element size is important for noise elimination without damaging the objects of interest.

\section{Watershed transformation}

In watershed segmentation an image is regarded as a topographic landscape with ridges and valleys. The elevation values of the landscape are typically defined by the gray values of the respective pixels or their gradient magnitude. In an image the gradient magnitude for watershed transformation is interpreted as a topographic surface. Watershed transformation disintegrates an image completely by assigning each pixel either to a watershed or to a region. Good estimation of gradients of the image plays an important role in complete division of an image using watershed transformation. But the gradient magnitude is very much sensitive to noise of an image. Due to this, the result obtained from the watershed transformation is affected by the background noise and generate an over-segmented image. Undersegmented image is also produced by edges with low contrast which generate small magnitude gradients. This lead to merged distinct regions erroneously. Watershed principle is used for segmentation by adopting different approaches. First the object needs to be marked and grows using watershed transformation. Here, local minima of image gradient is chosen as markers which leads to over segmentation and later followed by merging of the region. Watershed transformation based on the marker, chooses a specific marker position either defined by the user or determined using automatic morphological operators or other ways $(19,20,25)$.

\section{Marker-Controlled} segmentation

watershed

In image processing the most difficult task is to segregate the touching objects in an image using image processing operations. In such types of problem watershed algorithm is applied. The watershed transformation on an image determines catchment basins along with ridge lines by considering it as a surface with dark pixels as low and light pixels as high. The conventional way for determining the number and approximate region's location produce by the watersheds technique involved in modifying the homotopy of the function where the algorithm is applied. This is done through mathematical morphology operation or geodesic reconstruction through which the function is modified and minima can be established by the external function. The catchment basins which are not marked, are filled using morphological reconstruction and converted to non-minima plateaus that does not produce specific regions while calculating watersheds. Therefore, segmentation of the image using this technique works well for foreground and background identification as well as segregation of touching or overlapping objects in an image. The image obtained after watershed segmentation segregates the overlapping objects and each object is labelled individually (21). In this research work, we have taken images of 
piggeries and performed segmentation on those images, and labelled each pig in the image accurately and total number of objects are determined.

\section{Experiments}

The experimental datasets of piggery are collected from research papers (15). Maximum images are taken from top view so that each pig are of similar size. We have considered images of both overlapping as well as non-overlapping of objectsto verify the algorithm. First, we have considered a color image which is then split into RGB channels i.e red channel, green channel and blue channel. Green channel is chosen for further processing as the objects in an image are clearly visible in green channel due to its high contrast. Three types of thresholding techniques such as manual thresholding, Otsu thresholding, and adaptive thresholdng are used in this paper. The similarity of these thresholding algorithms as compared to ground truth is calculated based on jaccards coefficient similarity index (22). The mathematical expression of this index is defined as follows

jaccards $=\frac{\left\|B W_{1} \cap B W_{2}\right\|}{\left\|B W_{1} \cup B W_{2}\right\|}$,

where $B W_{1}$ denotes the segmented binary image using thresholding techniques and $B W_{2}$ denotes the ground truth binary image. Both quantitative and qualitative analysis is performed on the resultant images obtained from thresholding techniques. The mean similarity scores and execution time of each thresholding technique are determined and choose the best thresholding technique based on the two parameters.

Followed by morphological processing in order to remove the noise from the threshold images. In morphological filtering process, first the filling of holes in binary image is performed. Later, followed by morphological opening on the binary image using the structuring element of radius 10 pixels. The objects present in an image may be either in overlapping and non-overlapping structure. In case of non overlapping the counting of objects is simply done by labelling the segmented objects after threshold. But in case of overlapping objects, markers controlled watershed segmentation is used to segregate the touching objects so that the total number of objects can be calculated accurately. To determine whether the image have overlapping objects or not, we calculate the area of each contour and average of the areas. Mathematically, we calculated $C=\frac{\text { area }}{\text { avg area }}$, if this returns integer value greater than or equal to ${ }^{2}$ then it is consider to be image with overlapping objects otherwise image have non overlapping objects. This algorithm works under the assumption that images are taken from top view and objects are at equal distance from the camera so that it may be of the same sizes. Finally, each object in the image is counted by giving a unique numeric label. This experiment was conducted on Windows 8 operating system using Matlab $2017 b$.

\section{Results and Discussion}

The quantitative as well as qualitative analysis of three thresholding technique is performed. The quantitative analysis of thresholding techniques such as Manual, Otsu and Adaptive based on the Jaccards similarity score and execution time is shown in table 1. Jaccards similarity is calculated between the ground truth segmented image and the segmented image obtain from the thresholding techniques. From the table it has been shown that jaccards similiarty score of Otsu threshold gives the highest similarity scores with a value of 0.9302 and the 
execution time of Otsu threshold is 2.4534 sec Out of this three technique, Adaptive threshold gives the least execution time of $1.7506 \mathrm{sec}$. But adaptive threshold gives extremely low similarity score value of 0.7426 . Even though adaptive threshold executes faster, the similarity score is less. So, we have considered Otsu threshold to be more efficient to use for further analysis.

Moreover, we have also performed qualitative analysis of these threshold techniques and it has been shown in figure 2. In this figure, we have considered three piggery images, the ground truth of the images is also shown in the figure. The results obtained from manual threshold is very much similar with ground truth, but the execution of this algorithm takes more time and is not reliable. In case of adaptive threshold, the segmented image does not generate good resulting images for pictures with non uniform illumination. Next followed by Otsu threshold which produce a similar image to ground truth segmented images. Otsu is simple to calculate and it also takes less time for segmentation. The segmented image obtained from Otsu has less sensitive to noise. Therefore, from quantitative as well as qualitative analysis of the thresholding techniques we conclude that Otsu provides good resulting segmented image.

Table.1 Jaccards similarity score and execution time of three thresholding techniques

\begin{tabular}{|l|l|l|l|l|l|}
\hline \multicolumn{3}{|c|}{ Jaccards similarity score } & \multicolumn{3}{c|}{ Execution time } \\
\hline Manual & Otsu & Adaptive & Manual & Otsu & Adaptive \\
\hline $\mathbf{0 . 9 0 6 8}$ & 0.9302 & 0.7426 & $12.1417 \mathrm{sec}$ & $2.4534 \mathrm{sec}$ & $1.7506 \mathrm{sec}$ \\
\hline
\end{tabular}

Fig.1. Framework of pig counting system

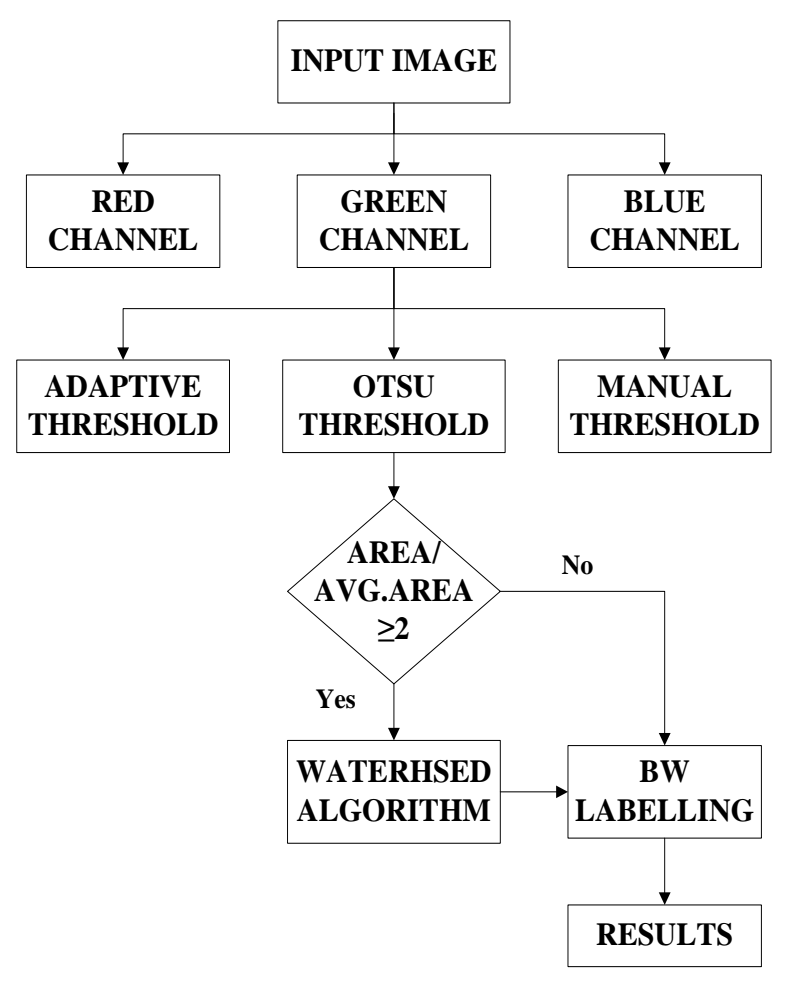


Fig.2 Three types of thresholding techniques and ground truth comparison
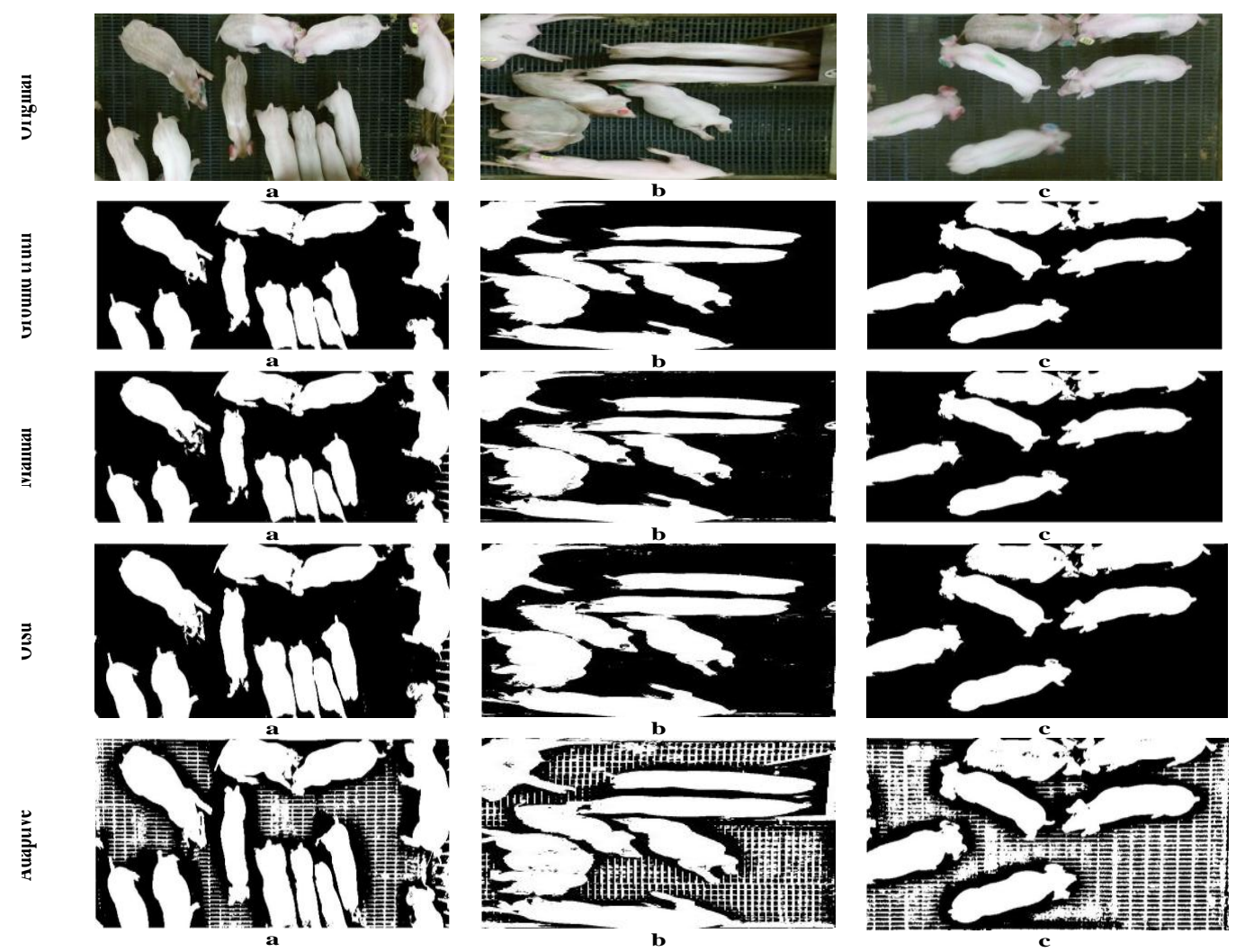

Fig.3 Illustration of Marker-Controlled watershed segmentation and counting process
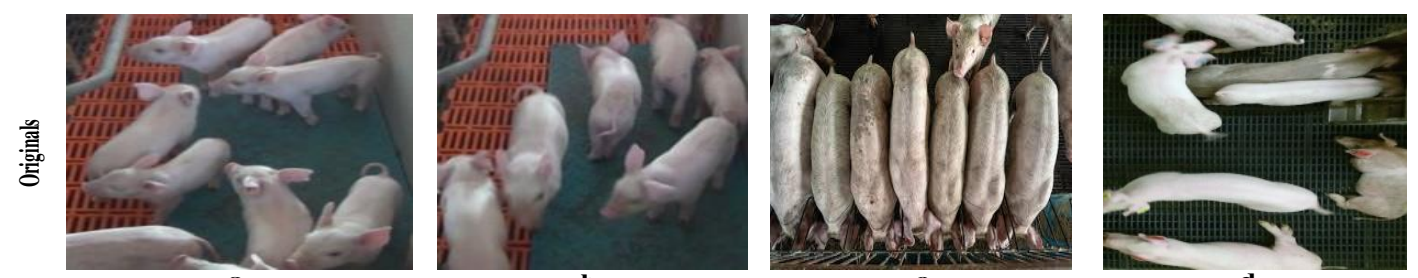

g
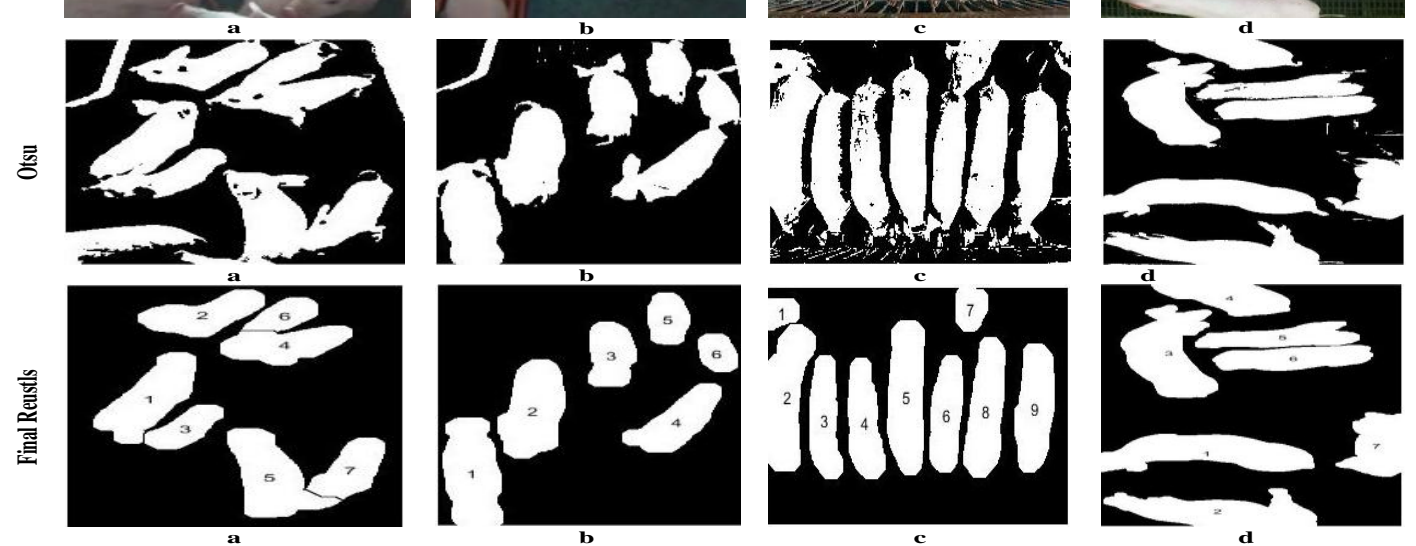
Counting of objects in an image has been illustrated in figure 3. In this, we have considered four piggery images. Then apply Otsu threshold on these images followed by morphological filtering. In all these four images, the objects (pig) are touching or overlapping with one another. To count these overlapping or touching objects individually, we considered Marker-controlled watershed segmentation process. The overlapping objects are segregated using Markercontrolled watershed segmentation and it is clearly shown in figure $3 \mathrm{a}$. Once the touching objects get segregated, all the objects are counted by giving the proper numeric label of each object. Hence, counting of pig has been successfully carried out using Otsu threshold and Marker-controlled watershed segmentation algorithm.

In conclusion this paper, we consider an image segmentation process for counting pigs from an image. We performed an analysis of different thresholding techniques to extract the foreground pixels. Comparative analysis of these thresholding techniques is performed based on similarity scores and execution time, where Otsu threshold is considered to be the best thresholding technique in terms of similarity and execution times. Further, the morphological filtering of filling the region and holes in an image is applied. Followed by opening filtering process in order to remove the unwanted noise from the image. Further, we determined the area of the contours of an object present in an image to predict the existence of overlapping objects in an image. Under the condition that if area of each object when divided by the average sum of contour area is greater than or equal to the interger ${ }^{2}$, then it is believed to have overlapping objects in an image. In case of overlapping objects. Marker controlled watershed segmentation is applied in order to distinguish each object uniquely. Then these objects are labelled with unique values so that the counting of objects can be done easily. Finally, we can conclude that objects counting can be done successfully using Marker-Controlled Watershed image segmentation algorithms.

\section{References}

1. Chauhan, A., Patel, B. H. M., Maurya, R., Kumar, S., Shukla, S., \& Kumar, S. (2016). Pig production system as a source of livelihood in Indian scenario: An overview. Int $J \quad S c i$ Environ Technol, 5(4), 2089-96.

2. https://www.agrifarming.in/commercialpig-farming-business-india.

3. Tianhao, Z., Yansen, L., \&Zhiyi, H. (2016). Applying image recognition and counting to reserved live pigs statistics. Computer Applications and Software, (12), 43.

4. Tian, M., Guo, H., Chen, H., Wang, Q., Long, C., \& Ma, Y. (2019). Automated pig counting using deep learning. Computers and Electronics in Agriculture, 163, 104840.

5. Zhan, Y., \& Zhang, G. (2019). An improved OTSU algorithm using histogram accumulation moment for ore segmentation. Symmetry, 11(3), 431.

6. Sangoi, L. F., Kessler, A. D. M., Neuenfeldt Júnior, A. L., Siluk, J. C. M., Ribeiro, A. M. L., \& Soliman, M. (2016). The use of artificial intelligence for the prediction of productivity parameters in swine

culture. PesquisaOperacional, 36(1), 6779.

7. Yadollahi, M., \&Procházka, A. (2011). Image Segmentation for Object Detection. In Proceedings of the 19th International Conference Technical Computing Prague 2011 (Vol. 129, pp. 1-12).

8. JU, M., CHUNG, Y., BAEK, H., CHUNG, Y., PARK, D., \& PARK, B. (2017). SEGMENTATION METHODS 
FOR A GROUP-HOUSED PIG MONITORING SYSTEM. Journal of Theoretical \& Applied Information Technology, 95(17).8

9. Marchant, J. A., \& Schofield, C. P. (1993). Extending the snake image processing algorithm for outlining pigs in scenes. Computers and electronics in agriculture, 8(4), 261-275.

10. Perner, P. (2001, May). Motion tracking of animals for behavior analysis. In International Workshop on Visual Form (pp. 779-786). Springer, Berlin, Heidelberg.

11. Lind, N. M., Vinther, M., Hemmingsen, R. P., \& Hansen, A. K. (2005). Validation of a digital video tracking system for recording pig locomotor behaviour. Journal of neuroscience methods, 143(2), 123-132.

12. Viazzi, S., Ismayilova, G., Oczak, M., Sonoda, L. T., Fels, M., Guarino, M.,... \&Berckmans, D. (2014). Image feature extraction for classification of aggressive interactions among pigs. Computers and Electronics in Agriculture, 104, 57-62.

13. Tu, G. J., Karstoft, H., Pedersen, L. J., \&Jørgensen, E. (2014). Segmentation of sows in farrowing pens. IET Image Processing, 8(1), 56-68.

14. Ahrendt, P., Gregersen, T., \&Karstoft, H. (2011). Development of a real-time computer vision system for tracking loose-housed pigs. Computers and Electronics in Agriculture, 76(2), 169174.

15. Kang, F., Wang, C., Li, J., \& Zong, Z. (2018). A Multiobjective Piglet Image Segmentation Method Based on an Improved Noninteractive GrabCut Algorithm. Advances in Multimedia, 2018.

16. Nilsson, M., Ardö, H., Åström, K., Herlin, A., Bergsten, C., \&Guzhva, O. (2014, August). Learning based image segmentation of pigs in a pen. In Visual observation and analysis of vertebrate and insect behavior-Workshop at the 22nd International Conference on Pattern Recognition (ICPR 2014), Stockholm, Sweden, August (pp. 24-28).

17. Heydari, M., Amirfattahi, R., Nazari, B., \& Rahimi, P. (2016). An industrial image processing-based approach for estimation of iron ore green pellet size distribution. Powder Technology, 303, 260-268.

18. JU, M., CHUNG, Y., BAEK, H., CHUNG, Y., PARK, D., \& PARK, B. (2017). SEGMENTATION METHODS FOR A GROUP-HOUSED PIG MONITORING SYSTEM. Journal of Theoretical \& Applied Information Technology, 95(17).

19. Kaur, M., \& Jindal, G. (2011). Medical Image Segmentation using Marker Controlled Watershed Transformation 1.

20. El Allaoui, A. (2012). Medical image segmentation by marker-controlled watershed and mathematical morphology. The International Journal of Multimedia \& Its Applications, 4(3), 1.

21. Rahman, M. S., \& Islam, M. R. (2013, February). Counting objects in an image by marker controlled watershed segmentation and thresholding. In 2013 3rd IEEE International Advance Computing Conference (IACC) (pp. 1251-1256). IEEE.

22. Niwattanakul, S., Singthongchai, J., Naenudorn, E., \&Wanapu, S. (2013, March). Using of Jaccard coefficient for keywords similarity. In Proceedings of the international multiconference of engineers and computer scientists (Vol. 1, No. 6, pp. 380-384).

23. Tu, G. J., Karstoft, H., Pedersen, L. J., \&Jørgensen, E. (2013). Foreground detection using loopy belief propagation. Biosystems engineering, 116(1), 88-96.

24. Guo, Y. Z., Zhu, W. X., Jiao, P. P., Ma, 
C. H., \& Yang, J. J. (2015). Multi-object extraction from top view group-housed pig images based on adaptive partitioning and multilevel thresholding segmentation. Biosystems Engineering, $135,54-60$.
25. Kornilov, A. S., \& Safonov, I. V. (2018). An overview of watershed algorithm implementations in open source libraries. Journal of Imaging, 4(10), 123.

\section{How to cite this article:}

Salam Jayachitra Devi and Manglem Singh, Kh. 2021. Counting Pig using Marker-Controlled Watershed Segmentation. Int.J.Curr.Microbiol.App.Sci. 10(01): 2069-2078.

doi: https://doi.org/10.20546/ijcmas.2021.1001.238 